\title{
Predictive Value of IHC4 Score for Pathological Response to Neoadjuvant Chemotherapy in Hormone Receptor-Positive Breast Cancer
}

\author{
Shereef Elsamany ${ }^{1,2 *}$, Soha Elmorsy ${ }^{3,4}$, Abdullah Alzahrani ${ }^{1}$, Ayman Rasmy ${ }^{5,6}$, \\ Waleed N Abozeed ${ }^{7,8}$, Amrallah A Mohammed ${ }^{1,6}$, Mohamed A Sherisher ${ }^{1,9}$, \\ Mohammed M Abbas ${ }^{10}$, Miral Mashhour ${ }^{11}$
}

\begin{abstract}
Purpose: This study aimed to explore the value of IHC4 in predicting pathological response after neoadjuvant chemotherapy in patients with hormonal receptor (HR)-positive breast cancer (BC). Materials and Methods: In this retrospective exploratory study, data for $68 \mathrm{HR}$-positive $\mathrm{BC}$ patients who received neoadjuvant chemotherapy were recorded. IHC4 scores were calculated based on estrogen receptors/progesterone receptors, Ki-67 and HER2 status. Logistic and ordinal regression analyses in addition to likelihood ratio test were used to explore associations of IHC4 scores and other clinico-pathological parameters with pathological complete response (pCR) and pathological stage. Results: Taking the 25th percentile as the cut-off, a lower IHC4 score was associated with an increased probability of pCR (low; $52.9 \%$ vs. High; $21.6 \%, \mathrm{OR}=4.1,95 \% \mathrm{CI}=1.28-13.16, p=0.018$ ) and a lower pathological stage $(\mathrm{OR}=\mathbf{3 . 9}, 95 \% \mathrm{CI}=\mathbf{1 . 3 4 - 1 1 . 3 3}, \mathrm{p}=\mathbf{0 . 0 1 2})$. When the IHC4 score was treated as a continuous variable, a lower score was again associated with an increased probability of $\mathrm{pCR}(\mathrm{OR}=1.010,95 \%$ $\mathrm{CI}=1.001-1.018, \mathrm{p}=0.025)$ and lower pathological stage $(\mathrm{OR}=1.009,95 \% \mathrm{CI}=1.002-1.017, \mathrm{P}=0.008)$. Lower clinical stage was associated with a better $\mathrm{pCR}$ rate that was of borderline significance $(\mathrm{P}=0.056)$. When clinical stage and IHC4 score were incorporated together in a logistic model, the likelihood ratio test gave a P-value of 0.004 after removal of the IHC4 score and 0.011 after removal of the stage, indicating a more significant predictive value of the IHC4 score for pCR. Conclusions: This study suggests that the IHC4 score can predict pathological response to neoadjuvant chemotherapy in HR-positive BC patients. This finding now needs to be validated in a larger cohort of patients.
\end{abstract}

Keywords: IHC4 score - neoadjuvant chemotherapy - pathological response - hormonal receptors - breast cancer

Asian Pac J Cancer Prev, 16 (17), 7975-7979

\section{Introduction}

Neoadjuvant chemotherapy is usually utilized in inoperable breast cancer $(\mathrm{BC})$ patients to allow for surgical resection and to facilitate breast conservative surgery in borderline candidates (Aapro 2011). However, compelling evidence displayed improved long term survival with the achievement of pathological complete response (pCR) (Cortazar et al., 2014). This raised a critical need for predictors of response to neoadjuvant chemotherapy to optimize therapeutic outcome. The Oncotype DX recurrence score, based on a 21-gene signature, has been proven to estimate the risk of recurrence in early hormone receptors (HR)-positive BC patients treated with adjuvant hormonal therapy (Dowsett et al., 2010). It has also been shown to predict response to neoadjuvant chemotherapy.
In Patients treated with neoadjuvant docetaxel, those with a high recurrence score were more likely to have pCR (Chang et al., 2008). In addition, pCR was linked with higher expression of proliferation-related genes and lower expression of estrogen receptor (ER)-related genes (Gianni et al., 2005). However, the cost and complexity of gene-based assays highlight the need for developing more simplified predictive tools.

Immune-histochemistry (IHC)-4 score was developed based on the assessment of four key proteins in breast cancer including ER, progesterone receptors (PR), HER2 and Ki-67 (Cuzick et al., 2011). In the adjuvant setting of ER-positive patients, IHC4 score was found to provide prognostic information similar to that provided by Oncotype DX-recurrence score (Cuzick et al., 2011). This score was further validated in the cohort of

${ }^{1}$ Oncology, King Abdullah Medical City, Makkah, Saudi Arabia, ${ }^{10}$ Pathology, ${ }^{4}$ Research, ${ }^{5}$ Oncology, ${ }^{11}$ Pathology, King Fahed Specialist Hospital, Dammam, ${ }^{8}$ Medical Oncology, King Khaled Hospital, King Saud University, Riyadh, Saudi Arabia, ${ }^{2}$ Oncology, Oncology Centre, Mansoura University, ${ }^{7}$ Clinical Oncology, Mansoura University Hospital, Mansoura, ${ }^{3}$ Pharmacology, Faculty of Medicine, ${ }^{9}$ Medical Oncology, National Cancer Institute, Cairo University, Cairo, ${ }^{6}$ Medical Oncology, Faculty of Medicine, Zagazig University, Zagazig, Egypt *For correspondence: shereefmohamad@yahoo.com 
patients involved in the tamoxifen exemestene adjuvant multinational (TEAM) study (Christiansen et al., 2012). However, the value of IHC4 score as a predictor of response to neoadjuvant chemotherapy is yet to be explored.

The present study aimed to explore the value of IHC4 score in predicting pathological response to neoadjuvant chemotherapy in HR-positive BC patients. This score may provide a simple non-costly approach to predict the benefit of neoadjuvant chemotherapy in this group of $\mathrm{BC}$ patients.

\section{Materials and Methods}

\section{Study population}

We screened female patients with histologically confirmed BC who received neoadjuvant chemotherapy and presented to three institutes in Saudi Arabia from September 2012 to September 2013. We included patients with locally advanced disease or those who required downsizing to be eligible for conservative breast surgery. We selected patients who have received at least four cycles of neoadjuvant chemotherapy; anthracycline-based, taxane-based or both. The type of chemotherapy was at the discretion of the treating physician. Patients must have available data of ER, PR, HER2 and Ki-67 at diagnostic biopsies and only those with HR-positive phenotype were included.

\section{Study design and procedures}

In this retrospective exploratory study, clinicopathological data was collected including prechemotherapy tumour size and lymph node (LN) status as assessed at baseline mammograms/ breast ultrasound. Clinical stage before starting chemotherapy was recorded according to the TNM staging system of the American Joint Committee on Cancer (AJCC), 7th edition. Tumour phenotype (ER, PR, HER2 status) and Ki-67 were recorded from reports of diagnostic biopsies taken before starting chemotherapy. HR- positivity was defined as ER and/or PR-positive tumour status and IHC4 score was calculated as stated below. Treatment data was recorded including type and number of chemotherapy cycles. Pathological tumour size and nodal status were recorded from pathological reports of definitive breast surgery. We defined pCR as the absence of any invasive carcinoma or carcinoma in situ in the breast or axillary LNs at the time of definitive breast surgery. Partial response (PR) and stable disease (SD) were defined according to Sataloff classification where PR corresponds to presence of evidence of therapeutic effect $(<50 \%$ or $>50 \%)$ while SD shows no evidence of therapeutic effect. Approvals from the institutional review board of contributing institutions were obtained before starting the study procedures.

\section{Calculation of IHC4 score}

As reported by Cuzick et al., 2011, ER was quantified using the $\mathrm{H}$-score, which was defined as the percentage of cells staining weakly plus two times the percentage of cells staining moderately plus three times the percentage of cells staining strongly. The variable ER 10 was obtained by dividing the $\mathrm{H}$-score by 30 to obtain a variable with a range of $0-10$. PR was scored as the percentage of cells staining positive with a cut-off of $10 \%$. PR10 was obtained by dividing this percentage by 10 . HER 2 status was assessed by IHC in addition to FISH confirmation in cases with $(2+)$ by IHC. Tumours that were $(3+)$ by IHC or (2+) with FISH-positive testing were considered HER2 positive. Ki67 was recorded according to the percentage of positively staining malignant cells. Manual reading of Ki67 was utilized and Ki67 values were divided by 2.5 for correction of higher values compared to computer-aided reading method that was used in generating the below equation. IHC4 score was then calculated as follows (Cuzick et al., 2011):

IHC4=94.7 x $\{-0.100$ ER10 -0.079 PR10+0.586 $\mathrm{HER} 2+0.240 \ln (1+10 \times \mathrm{Ki} 67)\}$

\section{Statistical analysis}

The data was analyzed using STATA version 11.0. Numeric data was presented as median as well as mean values \pm standard deviation (SD). Categorical variables were presented as percentages. A logistic and an ordinal regression models were constructed using the $\mathrm{PCR}$ and the pathological stage after surgery as the dependent variables respectively. The following parameters were entered into each model as explanatory variables in a univariate manner: absolute value of the IHC4 score, clinical stage at diagnosis, other clinico-pathological parameters (such as age, menopausal status at diagnosis and multicentricity), type and number of chemotherapy cycles $(<6 v s$. 6-8). In addition, categorical explanatory variables were created by setting two cut-off values to divide the IHC4 scores at the median and the $25 \%$ percentile. The latter variables were used to study the association of $\mathrm{pCR}$ and pathological stage after surgery with IHC4 score categories. In addition, the test of trend was used to assess the relation of IHC4 score with pathological stage. For the 1st dependent variable (pCR), a multivariate model was constructed that included all factors with significant association in univariate analysis. A likelihood ratio test was performed to illustrate the impact of removing each variable from the multivariate model. A two-sided alpha level of $<0.05$ was considered significant for all comparisons.

\section{Results}

We screened 241 BC patients who received neoadjuvant chemotherapy in the contributing institutes within the above specified period. Data of ER/PR, HER2 status at diagnostic biopsies was available for all patients while results of Ki67 were available in 101 patients and only 68 of them were HR-positive. Among those 68 patients, infiltrating duct carcinoma was the predominant pathological type $(91.2 \%), 25 \%$ were younger than 40 years and $63.2 \%$ were premenopausal at diagnosis. T4 tumours and clinical stage III were found in $19.1 \%$ and $63.2 \%$ of patients respectively. Only 3 patients with stage I disease who initially refused surgery were included. They have agreed later to go for definitive surgery after receiving primary chemotherapy course. Noteworthy, two thirds of our patients $(67.6 \%)$ had luminal B subtype 
IHC4 Score Predictive Value for Response to Neoadjuvant Chemotherapy in Hormone Receptor-Positive Breast Cancer

with either Ki67 expressed in $>14 \%$ of tumour cells or ER+/HER2+ irrespective of Ki67 level. The majority of patients received both anthracycline and taxanebased chemotherapy regimens (85.3\%) (3-4 cycles of anthracycline-based followed by 3-4 cycles of taxane-

Table 1. Characteristics of Study Population

\begin{tabular}{|c|c|}
\hline Parameters & $\mathrm{n}(\%)$ \\
\hline \multicolumn{2}{|l|}{ Age at diagnosis } \\
\hline$\leq 40$ & $16(23.5)$ \\
\hline$>40$ & $52(76.5)$ \\
\hline \multicolumn{2}{|l|}{ Menopause } \\
\hline Premenopausal & $43(63.2)$ \\
\hline Postmenopausal & $25(36.8)$ \\
\hline \multicolumn{2}{|l|}{ Pathology } \\
\hline Infiltrating duct carcinoma & $62(91.2)$ \\
\hline Lobular carcinoma & $6(8.8)$ \\
\hline \multicolumn{2}{|l|}{ Grade } \\
\hline 1 & $4 \quad(5.9)$ \\
\hline 2 & $44(64.7)$ \\
\hline 3 & $20(29.4)$ \\
\hline \multicolumn{2}{|l|}{ Multicentric tumours } \\
\hline Yes & $14(20.6)$ \\
\hline No & $54(79.4)$ \\
\hline \multicolumn{2}{|l|}{ Lymphovascular invasion } \\
\hline Yes & $30(44.1)$ \\
\hline No & $38(55.9)$ \\
\hline \multicolumn{2}{|l|}{ Clinical stage } \\
\hline I & 3 (4.4) \\
\hline II & $22(32.4)$ \\
\hline IIIA & $28(41.2)$ \\
\hline IIIB & $15(22.0)$ \\
\hline \multicolumn{2}{|c|}{ Tumour size before chemotherapy } \\
\hline $\mathrm{T} 1$ & $5(7.4)$ \\
\hline $\mathrm{T} 2$ & $23(33.8)$ \\
\hline T3 & $27(39.7)$ \\
\hline $\mathrm{T} 4$ & $13(19.1)$ \\
\hline \multicolumn{2}{|l|}{ Progesteron receptors } \\
\hline Positive & $57(83.8)$ \\
\hline Negative & $11(16.2)$ \\
\hline \multicolumn{2}{|l|}{ Ki-67 expression } \\
\hline$\leq 14 \%$ & $22(32.4)$ \\
\hline$>14 \%$ & $46(67.6)$ \\
\hline Parameters & $\mathrm{n}(\%)$ \\
\hline \multicolumn{2}{|l|}{ HER2 } \\
\hline Negative & $54(79.4)$ \\
\hline Positive & $14(20.6)$ \\
\hline \multicolumn{2}{|l|}{ Type of chemotherapy } \\
\hline Anthracycline and taxan & $58(85.3)$ \\
\hline Anthracycline or taxan & $10(14.7)$ \\
\hline \multicolumn{2}{|l|}{ No of chemotherapy cycles } \\
\hline$<6$ & $9(13.2)$ \\
\hline $6-8$ & $59(86.8)$ \\
\hline \multicolumn{2}{|l|}{ Response to chemotherapy } \\
\hline Complete response & $20(29.4)$ \\
\hline Partial response & $31(45.6)$ \\
\hline Stable disease & $14(20.6)$ \\
\hline Progressive disease & $3(4.4)$ \\
\hline \multicolumn{2}{|l|}{ Pathological stage } \\
\hline 0 & $20(29.4)$ \\
\hline I & $8(11.8)$ \\
\hline II & $18(26.5)$ \\
\hline IIIA & $11(16.2)$ \\
\hline IIIB & $11(16.2)$ \\
\hline
\end{tabular}

based chemotherapy) (Table 1). All HER2-positive patients received trastuzumab combined with taxanebased chemotherapy.

In our cohort, pCR was found in $29.4 \%$ of patients while PR, SD and progressive disease were encountered in $45.6 \%, 20.6 \%$ and $4.4 \%$ of patients respectively. Pathological stages I and II were found in $11.8 \%$ and $26.5 \%$ of patients respectively (Table 1 ). The distribution of IHC4 score in the study group is shown in (Figure 1) showing relatively symmetrical distribution (skewness = 0.138 ) and similarity to the Gaussian distribution (kurtosis $=-0.567)$. The median value and the $25^{\text {th }}$ percentile of IHC4 score were (-10.79) and (-58.28) respectively, while the mean value \pm SD was $(-10.68 \pm 67.77)$. When the $25^{\text {th }}$ percentile was utilized as the cut-off, lower IHC4 score was associated with an increased probability of having pCR (low; $52.9 \%$ vs. High; 21.6\%, OR=4.1, 95\% $\mathrm{CI}=1.28-13.16, \mathrm{p}=0.018)$ (Table 2) as well as lower pathological stage $(\mathrm{OR}=3.9,95 \% \mathrm{CI}=1.34-11.33$, $\mathrm{p}=0.012$ ) (Table 3). Likewise, taking the median value as the cut-off, patients with lower IHC4 score were more likely to have pCR compared to those with higher score, however, the difference was not statistically significant (38.2\% vs. $20.6 \%$ respectively, $\mathrm{OR}=2.4,95 \% \mathrm{CI}=0.81$ $7.04, \mathrm{p}=0.115$ ) (Table 2) and were more likely to have lower pathological stage $(\mathrm{OR}=3.18,95 \% \mathrm{CI}=1.31-7.71$,

Table 2. Relation of IHC4 Score with Pathological Complete Response (pCR)

\begin{tabular}{|c|c|c|c|c|}
\hline & $\begin{array}{l}\mathrm{pCR} \\
\mathrm{n}(\%)\end{array}$ & $\begin{array}{c}\text { No pCR } \\
\text { n }(\%)\end{array}$ & $\mathrm{OR}^{\mathrm{a}}\left(95 \% \mathrm{CI}^{\mathrm{b}}\right)$ & $\mathrm{P}$ \\
\hline \multicolumn{5}{|c|}{ IHC4 (25 ${ }^{\text {th }}$ percentile $)$} \\
\hline Low $(n=17)$ & $9(52.9)$ & $8(47.1)$ & \multirow[t]{2}{*}{$4.1(1.28-13.16)$} & \multirow[t]{2}{*}{0.018} \\
\hline $\operatorname{High}(n=51)$ & $11(21.6)$ & $40(78.4)$ & & \\
\hline \multicolumn{5}{|l|}{ IHC4 (median) } \\
\hline $\operatorname{Low}(n=34)$ & $13(38.2)$ & $21(61.8)$ & \multirow[t]{2}{*}{$2.4(0.81-7.04)$} & \multirow[t]{2}{*}{0.115} \\
\hline $\operatorname{High}(n=34)$ & 7 (20.6) & $27(79.4)$ & & \\
\hline Mean IHC4 score & -40.1 & 1.58 & - & - \\
\hline$\pm \mathrm{SD}^{\mathrm{c}}$ & \pm 78.88 & \pm 59.24 & & \\
\hline Median IHC4 scor & re -47.27 & 2.13 & - & 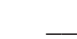 \\
\hline
\end{tabular}

a OR, odds ratio; ${ }^{\mathrm{b}} \mathrm{CI}$, confidence interval; $\mathrm{c} \mathrm{SD}$, standard deviation

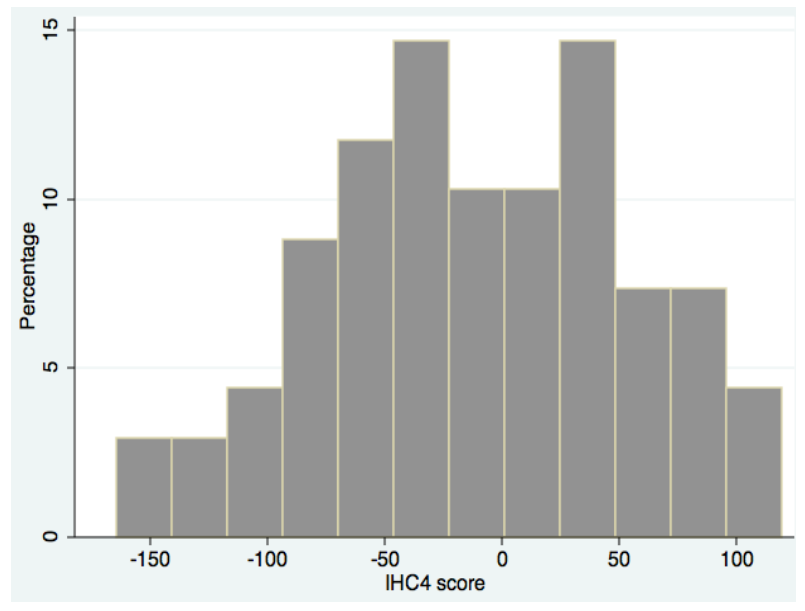

Figure 1. Histogram of IHC4 Score Distribution in the Study Group 
Table 3. Relation of IHC4 Score with Pathological Stage After Surgery

\begin{tabular}{|c|c|c|c|c|c|c|c|}
\hline & \multicolumn{6}{|c|}{ Pathological stage } & \multirow[b]{2}{*}{ p-value } \\
\hline & $\begin{array}{c}0 \\
\mathrm{n}(\%)\end{array}$ & $\begin{array}{c}\mathrm{I} \\
\mathrm{n}(\%)\end{array}$ & $\begin{array}{c}\text { II } \\
\text { n }(\%)\end{array}$ & $\begin{array}{c}\text { IIIA } \\
\text { n }(\%)\end{array}$ & $\begin{array}{c}\text { IIIB } \\
\mathrm{n}(\%)\end{array}$ & $\begin{array}{c}\mathrm{OR}^{\mathrm{a}} \\
\left(95 \% \mathrm{CI}^{\mathrm{b}}\right)\end{array}$ & \\
\hline \multicolumn{8}{|l|}{ IHC4 $\left(25^{\text {th }}\right.$ percentile $)$} \\
\hline Low $(n=17)$ & $9(52.9)$ & $3(17.6)$ & $3(17.6)$ & $1(5.9)$ & $1(5.9)$ & 3.9 & \\
\hline High $(\mathrm{n}=51)$ & $11(21.6)$ & $5(9.8)$ & $15(29.4)$ & $10(19.6)$ & $10(19.6)$ & $(1.34-11.33)$ & 0.012 \\
\hline \multicolumn{8}{|l|}{ IHC4 (median) } \\
\hline Low $(n=34)$ & $13(38.2)$ & $7(20.6)$ & $6(17.6)$ & $5(14.7)$ & $3(8.8)$ & 3.18 & \\
\hline High $(n=34)$ & 7 (20.6) & $1(2.9)$ & $12(35.3)$ & $6(17.6)$ & $8(23.6)$ & $(1.31-7.71)$ & 0.01 \\
\hline Mean IHC4 score $\pm \mathrm{SD}^{\mathrm{c}}$ & $\begin{array}{c}-40.1 \\
\pm 78.88\end{array}$ & $-28.08 \pm 63.88$ & $\begin{array}{c}11.74 \\
\pm 57.93\end{array}$ & $\begin{array}{c}-20.05 \\
\pm 48.02\end{array}$ & $\begin{array}{c}28.17 \\
\pm 58.69\end{array}$ & & \\
\hline Median IHC4 score & -47.27 & -43.33 & 30.09 & -11.32 & 21.49 & - & {[} \\
\hline
\end{tabular}

${ }^{\mathrm{a}} \mathrm{OR}$, odds ratio; ${ }^{\mathrm{b}} \mathrm{CI}$, confidence interval; ${ }^{\mathrm{c}} \mathrm{SD}$, standard deviation

$\mathrm{p}=0.010)$ (Table 3).

Noteworthy, the median and mean IHC4 scores were much lower in patients who achieved pCR compared to those with no pCR (median; -47.27 vs. 2.13 , mean \pm SD; $-40.1 \pm 78.88$ vs. $1.58 \pm 59.24$, respectively) (table 2). Likewise, patients with pathological stage I had a much lower IHC4 score compared to those who had stage IIIB post chemotherapy (median; -43.33 vs. 21.49 , mean \pm SD; $-28.09 \pm 63.89$ vs. $28.17 \pm 58.69$ respectively) (table 3 ). When IHC4 score was treated as a continuous variable, lower score was significantly associated with an increased probability of $\mathrm{pCR}(\mathrm{OR}=1.01,95 \% \mathrm{CI}=1.001-1.018$, $\mathrm{p}=0.025)$ and lower pathological stage $(\mathrm{OR}=1.009,95 \%$ $\mathrm{CI}=1.002-1.017, \mathrm{P}=0.008)$. By doing test of trend, $\mathrm{IHC} 4$ score was significantly correlated with the pathological stage, $\mathrm{p}=0.034$.

Lower clinical stage was associated with a better $\mathrm{pCR}$ rate that was of borderline significance $(\mathrm{p}=0.056)$, while no significant associations were found between other parameters and pCR rate. Accordingly, the clinical stage and the IHCR score were incorporated in a bivariate logistic model. Using the likelihood ratio test, removal of IHC4 score from the bivariate model gave a chi square value $\left(\mathrm{LR}-\chi^{2}\right)$ of $8.19, \mathrm{p}=0.004$. After removal of clinical stage from the model, $\mathrm{LR}-\chi^{2}$ was $11.1, \mathrm{p}=0.011$ indicating a more powerful predictive value of IHC4 score for pCR.

\section{Discussion}

In current clinical practice, neoadjuvant chemotherapy is commonly used for the treatment of breast cancer patients (Telli 2013). In a pooled analysis of data of 12 trials including almost 12,000 patients, achievement of pCR was associated with long term survival benefit in those with HER2-positive, triple negative $\mathrm{BC}$ in addition to patients with luminal-B disease (Cortazar et al., 2014). In addition, patients with lower pathological stage after neoadjuvant chemotherapy were associated with a better survival outcome (Kim et al., 2013). Despite the low pCR rates following neoadjuvant chemotherapy in HR-positive breast cancer (Telli, 2013), certain HR-positive patients still have appreciated benefit from adjuvant chemotherapy (Dowsett et al., 2010). This data raises the need to explore new predictive tools to optimize the use of neoadjuvant chemotherapy in HR-positive patients to select patients who are more likely to benefit from it.

The percentage of pCR in our study was high compared to previous reports that demonstrated low response rate in HR- positive tumours ranging from 2-10\% (Colleoni et al., 2004; Kaufmann et al., 2012). Our cohort has several characteristics that may explain this unexpected high response rate to chemotherapy. Two thirds of patients were premenopausal and the majority received 6-8 cycles of both anthracycline and taxan-based chemotherapy. Two thirds of patients had luminal B subtype which is more likely to respond to chemotherapy (Lonning, 2012).

In the present study involving HR-positive patients, low IHC4 score was associated with better pCR and lower pathological stage after neoadjuvant chemotherapy. Noteworthy, IHC4 score and clinical stage had additive predictive powers for $\mathrm{pCR}$, however, the score was more predictive for $\mathrm{pCR}$ which highlights its promising role in this regard. Cuzick et al., (2011) demonstrated improved survival outcome in patients with lower IHC4 score in the adjuvant setting. It is to be noted, however, that lower IHC4 score is linked with higher HR- positivity which is usually associated with favourable prognostic outcome but lower benefit from chemotherapy. Several reports have suggested that ER-negative tumours derive more benefit from neoadjuvant chemotherapy than their ER-positive counterparts (Barrios et al., 2009). In addition, the degree of response to chemotherapy was linked with degree of HR-positivity (Colleoni and Montagna, 2012). Yet, the recent 2012 overview analysis of Early Breast Cancer Trialist Collaborative Group displayed that chemotherapy benefit is independent of ER receptor status (Peto et al., 2012). In view of this, the higher response to neoadjuvant chemotherapy linked with lower IHC4 score among our cohort needs to be taken with caution.

In HR-positive patients, chemotherapy type and intensity seems to be of paramount value. Several reports displayed improved pCR in HR-positive patients with prolonged duration of chemotherapy (Colleoni and Montagna, 2012). In NSABP-B 27 trial, adding docetaxel after 4 cycles of doxorubicin and cyclophoshamide chemotherapy was associated with increased pCR in HR-positive patients (Bear et al., 2003). Furthermore, in a pooled analysis of the German neoadjuvant chemotherapy trials including more than 3000 women, the association of increased number of chemotherapy courses with $\mathrm{pCR}$ 
IHC4 Score Predictive Value for Response to Neoadjuvant Chemotherapy in Hormone Receptor-Positive Breast Cancer

was more pronounced in HR-positive compared to HRnegative patients (Minckwitz et al., 2011).

Among HR-positive patients, certain high-risk subgroups seem to derive increased benefit from intensified neoadjuvant chemotherapy such as ERpositive/HER2-positive tumours or ER-positive tumours with a high proliferation rate. Penault et al. (2009) showed improved outcome with the addition of taxanes to 3 cycles of FEC compared to 6 cycles of FEC alone in ER-positive patients with elevated Ki67 $>20 \%$. Similarly, in HER2positive tumours, trastuzumab-containing chemotherapy produced high pCR in the range of $30-40 \%$ which is double that reported in HER2-negative patients (Gianni et al., 2010; Untch et al., 2011).

It is to be noted that high $\mathrm{Ki}-67$ expression was predominant among our patients and $20 \%$ of our cohort, who were HER2-positive, received trastuzumabcontaining chemotherapy. Chemotherapy type and intensity may be linked with this unexpected improved response to neoadjuvant chemotherapy in those patients with lower IHC4 score. Our study including mainly high-risk, HR-positive patients may raise the issue of the interplay between the type of treatment and tumour biology in formulating the overall outcome of HR-positive patients. It seems that more intense chemotherapy may translate the favourable prognosis of lower IHC4 score into better response to neoadjuvant chemotherapy as well.

Many questions are still to be answered. Proper utilization of IHC4 score to stratify patients according to differential response to neoadjuvant chemotherapy, integration of clinical characteristics with this biomarker score and how to apply the score for the individual patient in routine practice, are still open fields that need further work to enhance the utility of this score. In conclusion, lower IHC4 score seems to be associated with improved response to neoadjuvant chemotherapy in a group of predominantly high risk, HR-positive patients. This finding needs to be confirmed in a larger cohort of patients to validate the role of IHC4 score as a predictive tool in this context.

\section{References}

Aapro M (2011). Neoadjuvant therapy in breast cancer: can we define its role? The Oncologist, 6, 36-39.

Barrios CH, Sampaio C, Vinholes J, et al (2009). What is the role of chemotherapy in estrogen receptor-positive, advanced breast cancer? Ann Oncol, 20, 1157-62.

Bear HD, Anderson S, Brown A, et al (2003). The effect on tumour response of adding sequential preoperative docetaxel to preoperative doxorubicin and cyclophosphamide: preliminary results from National Surgical Adjuvant Breast and Bowel Project Protocol B-27. J Clin Oncol, 21, 4165-74.

Chang JC, Makris A, Gutierrez MC, et al (2008). Gene expression patterns in formalin-fixed, paraffin-embedded core biopsies predict docetaxel chemosensitivity in breast cancer patients. Breast Cancer Res Treat, 108, 233-40.

Christiansen J, Bartlett J, Gustavson M, et al (2012). Validation of IHC4 algorithms for prediction of risk of recurrence in early breast cancer using both conventional and quantitative IHC approaches. J Clin Oncol, 30, 517.

Colleoni M, Montagna E (2012). Neoadjuvant therapy for ERpositive breast cancers. Ann Oncol, 23, 243-48.
Colleoni M, Viale G, Zahrieh D, et al (2004). Chemotherapy is more effective in patients with breast cancer not expressing steroid hormone receptors: a study of preoperative treatment. Clin Cancer Res, 10, 6622-8.

Cortazar P, Zhang L, Untch M, et al (2014). Pathological complete response and long-term clinical benefit in breast cancer: the CTNeoBC pooled analysis. Lancet, 384, 164-72.

Cuzick J, Dowsett M, Pineda S, et al (2011). Prognostic value of a combined estrogen receptor, progesterone receptor, $\mathrm{Ki}-67$, and human epidermal growth factor receptor-2 immunohistochemical score and comparison with the Genomic Health recurrence score in early breast cancer. $J$ Clin Oncol, 29, 4273-8.

Dowsett M, Cuzick J, Wale C, et al (2010). Prediction of risk of distant recurrence using the 21-gene recurrence score in node-negative and node-positive postmenopausal patients with breast cancer treated with anastrozole or tamoxifen: a TransATAC study. J Clin Oncol, 28, 1829-34.

Gianni L, Eiermann W, Semiglazov V, et al (2010). Neoadjuvant chemotherapy with trastuzumab followed by adjuvant trastuzumab versus neoadjuvant chemotherapy alone, in patients with HER2-positive locally advanced breast cancer (the NOAH trial): a randomised controlled superiority trial with a parallel HER2-negative cohort. Lancet, 375, 377-84.

Gianni L, Zambetti M, Clark K, et al (2005). Gene expression profiles in paraffin-embedded core biopsy tissue predict response to chemotherapy in women with locally advanced breast cancer. J Clin Oncol, 23, 7265-77.

Kim MM, Allen P, Gonzalez-Angulo AM, et al (2013). Pathologic complete response to neoadjuvant chemotherapy with trastuzumab predicts for improved survival in women with HER2-overexpressing breast cancer. Ann Oncol, 24, 1999-2004.

Kaufmann M, Von Minckwitz G, Mamounas EP, et al (2012). Recommendations from an International consensus conference on the current status and future of neoadjuvant systemic therapy in primary breast cancer. Ann Surg Oncol, 19, 1508-16.

Lonning PE (2012). Poor-prognosis estrogen receptor- positive disease: present and future clinical solutions. Ther $\mathrm{Adv}$ Med Oncol, 4, 127-37.

Penault-Llorca F, Andre F, Sagan C, et al (2009). Ki67 Expression and docetaxel efficacy in patients with estrogen receptor-positive breast cancer. J Clin Oncol, 27, 2809-15.

Peto R, Davies C, Godwin J, et al (2012). Comparisons between different polychemotherapy regimens for early breast cancer meta-analysis of long-term outcome among 100.000 women in 123 randomised trials. Lancet, 379, 432-44.

Telli ML (2013). Insight or confusion: survival after responseguided neoadjuvant chemotherapy in breast cancer. J Clin Oncol, 31, 3613-5.

Untch M, Fasching PA, Konecny GE, et al (2011). Pathologic complete response after neoadjuvant chemotherapy plus trastuzumab predicts favourable survival in human epidermal growth factor receptor 2-overexpressing breast cancer: results from the TECHNO Trial of the AGO and GBG Study Groups. J Clin Oncol, 29, 3351-7.

Von Minckwitz G, Untch M, Nüesch E, et al (2011). Impact of treatment characteristics on response of different breast cancer phenotypes: pooled analysis of the German neoadjuvant chemotherapy trials. Breast Cancer Res Treat, 125, 145-56. 